\title{
Upregulation of mitogen-activated protein kinase in ganglioglioma
}

\author{
Beata Rak ${ }^{1}$, Stanisław Szlufik ${ }^{1}$, Wiesława Grajkowska ${ }^{2,3}$, Danuta Perek ${ }^{4}$, Bożena Dembowska-Bagińska ${ }^{4}$, \\ Iwona Filipek ${ }^{4}$, Paweł Daszkiewicz ${ }^{5}$, Paweł Włodarski ${ }^{1}$, Jarosław Jóźwiak ${ }^{1}$ \\ ${ }^{1}$ Department of Histology and Embryology, Center for Biostructure Research, Medical University of Warsaw, ${ }^{2}$ Department \\ of Experimental and Clinical Neuropathology, Mossakowski Medical Research Centre, Polish Academy of Sciences, \\ ${ }^{3}$ Department of Pathology, The Children's Memorial Health Institute, ${ }^{4}$ Department of Oncology, The Children's Memorial \\ Health Institute, ${ }^{5}$ Department of Neurosurgery, The Children's Memorial Health Institute, Warsaw, Poland
}

\begin{abstract}
Ganglioglioma $(G G)$ is a low-grade neoplasm, often associated with intractable epilepsy in pediatric patients. Available data suggest a relationship between GG and other glioneuronal lesions. So far, little is known about activation of kinases belonging to the mTor (mammalian target of rapamycin) pathway, although its upregulation is often found in brain tumors. Involvement of mTor kinase is responsible for excessive proliferation. In the current study we focused on the possible role of the Erk/Mapk (extracellular-signal-regulated kinase/mitogen-activated protein kinase) pathway in GG development. Eight GG tumors were resected from pediatric patients. Collected data reveal activation of proteins from the Erk pathway: Mek (extracellular regulated protein kinase kinase/mitogen-activated protein kinase kinase), Erk, Rsk1 (ribosomal $S 6$ kinase 1), Rheb (Ras homolog enriched in brain) and Msk1 (mitogen-and stress-activated protein kinase 1), as detected by the western blot method. Moreover, activation of other proteins upstream of mTor - IGF-1R $\beta$ (insulin-like growth factor $1 \beta$ receptor), INR $\alpha$ (insulin receptor $\alpha$ ), Akt/PKB (protein kinase B) - or downstream - 4E-BP1 (eukaryotic translation initiation factor 4E-binding protein 1) and rpS6 (ribosomal protein 56) - was also confirmed.
\end{abstract}

Key words: Akt, Erk, MAPK, ganglioglioma.

\section{Introduction}

Ganglioglioma (GG) is a non-malignant tumor, arising in the central nervous system and classified as G1 by the World Health Organization (WHO). The tumor consists of neoplastic ganglionic cells and a glial component, especially astrocytes $[4,18]$. GG affects mostly children and young adults, accounting for $0.4-2.1 \%$ of all brain tumors in this age group [6]. In children, the glial component typically resembles pilocytic as- trocytoma, with areas of microcystic changes and the presence of eosinophilic granular bodies (Fig. 1A). The ganglion cells are large, with amphophilic or eosinophilic cytoplasm and round, vesicular nuclei, and centrally located prominent nucleoli (Fig. 1B). Sometimes ganglion cells create binucleate forms. Ganglion cells are usually irregularly distributed. Focal lymphocytic aggregates are commonly seen. Immunohistochemically, the glial component displays strong expression of GFAP (Fig. 1C). GGs demonstrate 
immunoreactivity of neuronal and glial markers in appropriate cellular elements. Superficial perinuclear reaction for synaptophysin, which had been initially claimed to be tissue-specific for neuronal neoplasms, can be found in normal neurons and ganglion cells localized close to pathological sites [21,23]. Therefore, neoplastic ganglion cells in GG show intense immunoreactivity for synaptophysin as well as neurofilament protein (Fig. 1D). GG can develop almost in every area of the brain; however, there is a significant prevalence of GG occurrence in the supratentorial location, especially the temporal and frontal lobe. Treatment is based on total resection with disease-free 5-year survival over $80 \%[6,11,20]$. The most frequent symptom in patients with GG is epilepsy [25,22].

GGs reveal high expression of CD34, a stem cell marker, which occurs during early neurulation [10]. According to the study of Blumcke et al., CD34 is positive in $74 \%$ of GGs and has been correlated with S-100 protein immunoreactivity [3]. As far as tumorigenesis of ganglioglioma is concerned, which histologically is composed of cells similar to giant cells of tuberous sclerosis, i.e. balloon cells, it may be caused by excessive activation of mTOR (mammalian target of rapamycin). It is hypothesized that mTOR upregulation could be a result of somatic gene mutations occurring during development of the brain [7]. Furthermore, there are also data suggesting the possibility of polymorphism resistance in intron 4 and exon 41 of the TSC2 gene, as well as intron 13 and exon 14 of the TSC1 gene in GG. Moreover, only in the glial part of the tumor has polymorphism of intron 32 of the TSC2 gene been found [8]. However, Prayson et al. [22] did not find any mutation in the examined area of the genes.

mTOR kinase is known to be a central regulator of cellular state and processes such as autophagy, angiogenesis and proliferation. mTOR activity depends on triggering extracellular and intracellular signals: protein kinase B (PKB or Akt) and Erk (extracellular signal-regulated protein kinase or mitogen activated protein kinase, MAPK) signaling pathways. In case of excessive stimulation, mTOR may provoke tumorigenesis. On the other hand, if a cell lacks energy, oxygen or nutrients, proliferation is stopped by synthesis of inhibiting protein kinases. Both Akt and Erk pathways through the mediation of hamartin (or tuberous sclerosis complex-1 - TSC1) and tuberin (or tuberous sclerosis complex-2 - TSC2) act on mTOR. Rheb, belonging to the Ras superfamily, leads to phosphorylation of mTOR and increased activity of mTOR effectors: ribosomal protein S6 (rpS6) and eukaryote initiation factor-binding protein (4E-BP1). rpS6 protein is responsible for ribosome biogenesis and $4 \mathrm{E}-\mathrm{BP} 1$ is a regulator of protein translation $[2,13]$.

So far, little is known of the molecular pathogenesis of GG. Several reports suggest that the PI3K pathway may be the cause of hyperactivation of mTOR pathway in this tumor $[7,10,13]$. At the same time, upregulation of mTOR kinase is often found in brain tumors, especially associated with tuberous sclerosis $[9,14,15]$.

In the current study we decided to examine activation of Akt kinase as well as proteins belonging to the Erk pathway: mitogen-activated protein kinase kinase (MAPKK or Mek), the $90 \mathrm{kDa}$ ribosomal $\mathrm{S6}$ kinase (Rsk1), mitogen and stress activated protein kinase (Msk1) and Rheb, which act upstream of mTOR. Also, we focused on activation of the mTOR effectors rpS6 protein and 4E-BP1, in order to confirm or exclude mTOR involvement in the process of tumorigenesis.

\section{Material and methods}

Evaluated samples of tumors as well as control tissues were retrieved from the Department of Pathology, Children's Memorial Hospital in Warsaw, Poland. The research was performed on GGs resected from 8 patients during elective surgery. All cases of GG were classified as G1 by WHO criteria, but were arising in different parts of the brain, including the temporal lobe as well as the cerebellum (Table I). Control brain tissue was derived from patients operated on for epilepsy (periventricular region).

Clinical data reveal equal numbers of GG in male and female patients. The average age was 12.5 years. Almost in all cases (7 out of 8) patients presented with intractable epilepsy, which did not resolve in 3 cases. There exists significant prevalence of GG development in the temporal lobe of the brain.

\section{Western blot}

The first step of Western blot is electrophoretic separation of proteins. For this purpose, tissues were homogenized in a tissue grinder with RIPA lysis buffer $(0.1 \%$ SDS, $20 \mathrm{mM}$ Tris, pH 7.5, 1\% Nonidet P-40, $150 \mathrm{mM} \mathrm{NaCl}, 0.5 \%$ sodium deoxycholate, $1 \mathrm{mM}$ EDTA,) $1 \mathrm{mM}$ sodium orthovanadate and with $50 \mathrm{mM}$ sodium fluoride and supplemented with $1 \times \mathrm{Com}$ - 
Table I. Clinical data of patients with GG

\begin{tabular}{lccccccc}
\hline & $\begin{array}{l}\text { Grade } \\
\text { by WHO }\end{array}$ & Age (yrs) & Sex & Epilepsy & Localization of GG & Side & Epilepsy after surgery \\
\hline GG1 & 1 & 15 & F & + & Temporal lobe & Left & + \\
\hline GG2 & 1 & 6 & M & + & Temporal lobe & Left & - \\
\hline GG3 & 1 & 8 & M & + & Temporal lobe & Right & - \\
\hline GG4 & 1 & 15 & M & + & Temporal lobe & Left & + \\
\hline GG5 & 1 & 14 & M & + & Parietal lobe & Right & + \\
\hline GG6 & 1 & 9 & F & + & Temporal lobe & Left & - \\
\hline GG7 & 1 & 16 & F & - & Vermis of cerebellum & & - \\
\hline GG8 & 1 & 17 & F & + & Temporal lobe & Right & - \\
\hline
\end{tabular}

plete Protease Inhibitor Cocktail I (Sigma-Aldrich, St. Louis, MO). In order to minimize differences between samples in preparation procedure, all the samples were prepared in the same conditions, at the same time. Afterwards, all tumors and control brain lysates were stored at $-80^{\circ} \mathrm{C}$.

Obtained lysates of tissues $(10 \mu \mathrm{g})$ were pooled with the same amount of SDS PAGE. Prepared sam-
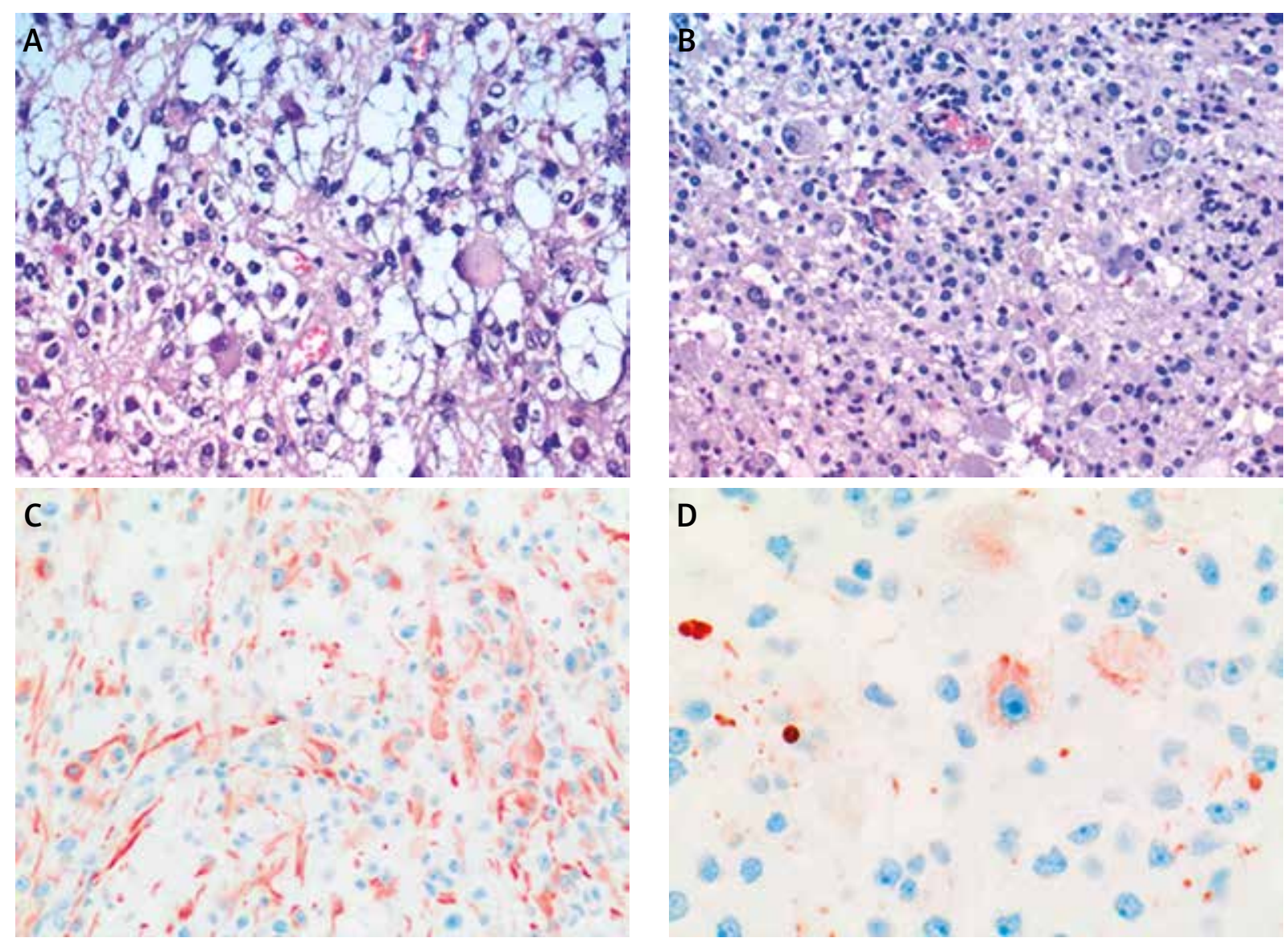

Fig. 1. A) Ganglioglioma. The glial component is similar to a pilocytic astrocytoma. H\&E staining. B) Large, atypical ganglion cells admixed with astrocytic glial component. Note granular eosinophilic bodies. H\&E staining. C) GFAP immunoreactivity of glial component. D) Neurofilament protein immunoexpression in ganglion cells. 
ples were warmed up to $90^{\circ} \mathrm{C}$ and microcentrifuged at $16,000 \mathrm{rpm}$. Afterwards we loaded lysates into any-kD gel (BIO-RAD Laboratories, Hercules, USA) and performed electrophoresis. After separation in an electric field, the blots were transferred onto PVDF membranes and blocked with $5 \%$ dry non-fat milk dissolved in TBST buffer $(0.05 \%$ Tween and Tris buffered saline). Presence of proteins in evaluated samples was detected and visually verified by staining with Ponceau S. The next step was membrane incubation with primary antibodies (mouse or rabbit) and secondary (HRP-conjugated). The final protein detection was performed by West Pico chemiluminescence substrate (Pierce, Rocford, IL), after washing in TBST buffer. Antibodies against phospho-4E-BP1 (Thr 37/46), phospho-IGF-I receptor $\beta$ (Tyr 1135/1136)/insulin receptor $\beta$ (Tyr1150/1151), phospho-Erk (Thr202/Tyr204), phospho-Akt (Ser 473), phospho-Rsk1 (Ser 380), phospho-Mek1/2 (Ser 221), phospho-S6 Ribosomal Protein (Ser 235/236), phospho-Msk1 (Thr 581) phospho-SHP2 and Rheb antibody were purchased from Cell Signaling Technology (Beverly, MA). Insulin Ra (N-20) antibody was obtained from Santa Cruz Biotechnology (Santa Cruz, CA).

\section{Results}

We demonstrated activation (phosphorylation on Ser 473) of Akt in all 8 patients, compared to negative reaction of control brain. Insulin receptor $\alpha$ expression was found in all examined samples. At the same time, we found strong activation of IGF-1R $\beta$ in 7 GG samples (Fig. 2).
Strong activation of Erk was found in all cases. Also, we studied activation of some kinases belonging to the Erk pathway, including Rsk1, Mek and Msk1. Phospho-Mek was found in 6 out of 8 samples. As far as Rsk1 is concerned, significant activation was confirmed in 5 out of 8 patients; additionally 1 tumor showed a weak signal of phospho-Rsk1. Msk1, acting downstream of Erk, was triggered in 7 out of 8 patients (weak activation in one case). Erk and Akt, by interaction with tuberous sclerosis complex (TSC1/TSC2) and Rheb, led to excessive activation of mTOR. A very strong signal of Rheb antibody was found in 7 out of 8 cases, and moderate in the remaining case (Fig. 3).

Then, we resolved to study both effectors of mTOR: 4E-BP1 and rpS6. Phosphorylated forms of ribosomal protein $\mathrm{S} 6$, responsible for protein translation and ribosome recruitment, were observed in all GG cases. Also, active form of 4E-BP1 was confirmed in 5 out of 8 samples (Fig. 4).

\section{Discussion}

GG often coexists with intractable, pharmacoresistant epilepsy. The same situation in observed with tuberous sclerosis, focal cortical dysplasia or hemimegalencephaly, which form a group of so-called mTORopathy. So far the only treatment is surgical resection, which both eliminates source of epilepsy and provides prevention of malignant transformation. Nevertheless, Curatolo and Moavero suggest in their review that therapy of rapamycin and everolimus are a promising treatment for mTORopathy-related epilepsy (including GG) in the future [8]. Only

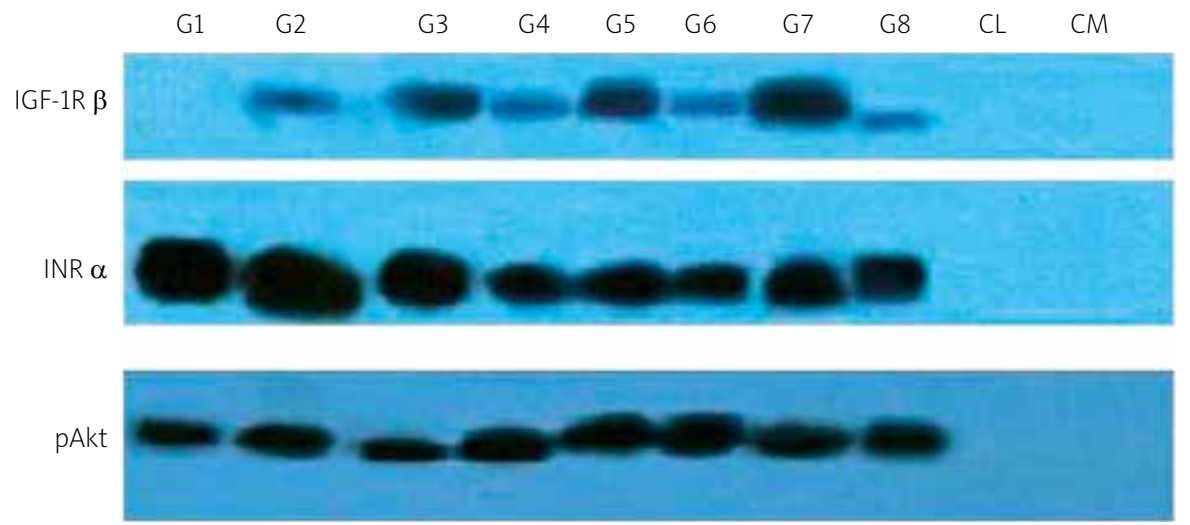

Fig. 2. Western blot showing activation of Akt kinase and two receptors potentially triggering Akt: IGF-IR $\beta$ and insulin receptor (IR) $\alpha$ in 8 GG cases (labeled G1-G8). CL - human control brain, CM - murine control brain. Equal protein loading was tested with $\alpha$-tubulin (not shown). 

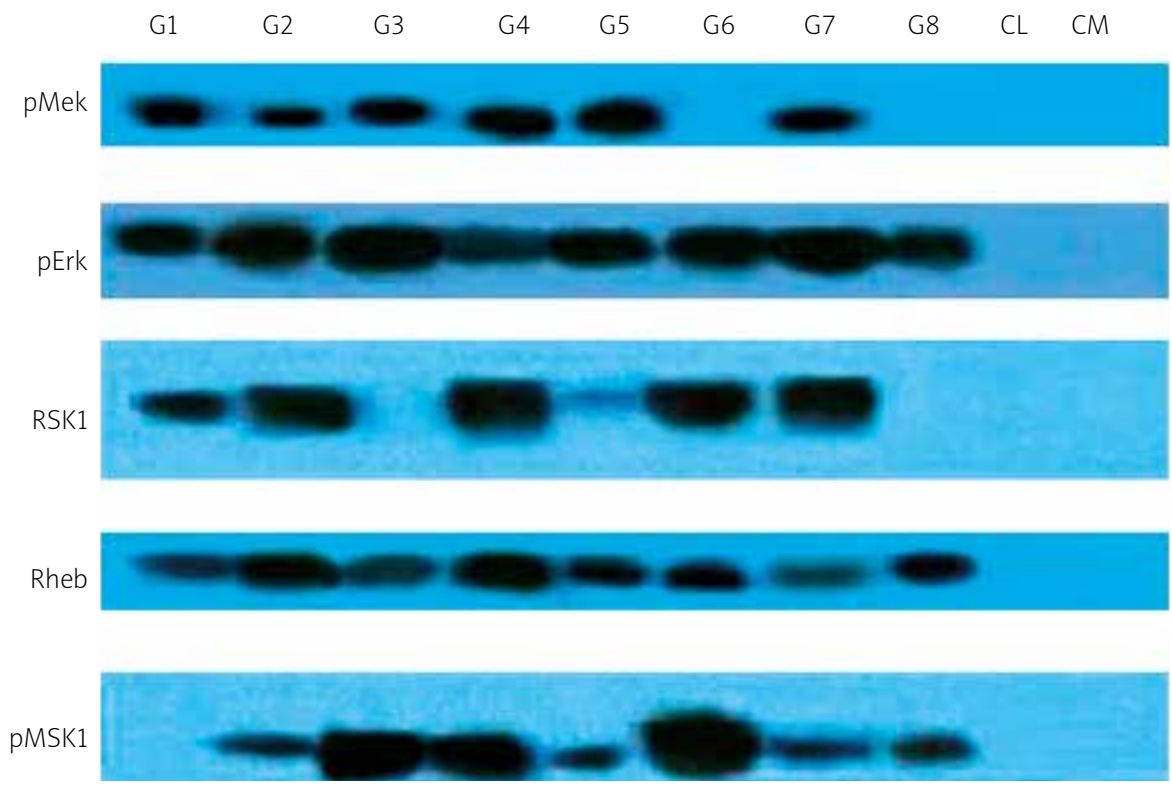

Fig. 3. Elements of the Erk pathway and their phosphorylation in the effect of activation in 8 GG tumors. The figure also shows Msk1, another kinase belonging to the family of MAPK kinases. CL - human control brain, CM - murine control brain. Equal protein loading was tested with $\alpha$-tubulin (not shown).
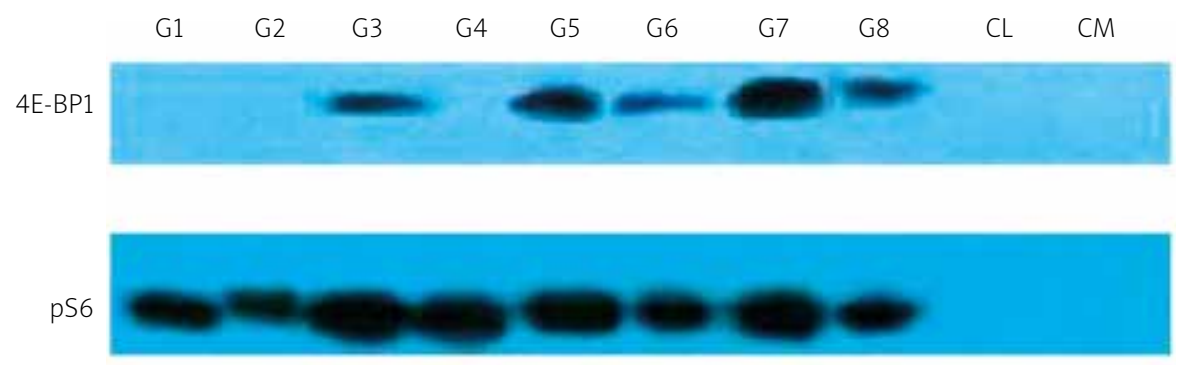

Fig. 4. Western blot of mTOR effectors in 8 GG tumors: 4E-BP1 and rpS6 are phosphorylated when upstream signals (e.g. Akt or Erk) potentiate mTOR activity. $\mathrm{CL}$ - human control brain, $\mathrm{CM}$ - murine control brain. Equal protein loading was tested with $\alpha$-tubulin (not shown).

limited data confirmed the role of proteins belonging to the PI3K/Akt pathway as a possible mechanism of tumorigenesis in GG. However, several studies suggest involvement of the PI3K/Akt signaling cascade in the pathogenesis of glioneuronal lesions, including focal cortical dysplasia $[5,19,24]$. In a recent report, Boer and colleagues revealed significant activation of PDK1, Akt, mTOR as well as rpS6 and 4E-BP1 in 9 cases of ganglioglioma [5]. In this regard, our study seems to be an extension of a larger group of earlier reports concerning activation of Akt, rpS6 and 4E-BP1 in GG.

Growth factors and insulin regulate mTOR activity by triggering the Akt pathway [12]. This may be the reason why in all GG cases with active Akt kinase also phosphorylated forms of insulin receptor $\alpha$ were con- firmed. Surprisingly, phospho-IGF-I receptor $\beta$ /insulin receptor $\beta$ was not found in all examined samples.

According to our knowledge, there exists only one publication which confirms occurrence of active Erk in two human GG cases. Aronica et al. suggest a link between function of Kv4.2 voltage-gated potassium channel and epilepsy in GG. Moreover, it has been shown that the Kv4.2 channel is one of the substrates for Erk [1]. We managed to confirm Erk activation in all evaluated samples.

Until now, other proteins of the Erk pathway, studied by our team - Mek, Rsk1, Msk1 and Rheb have never been evaluated. We have focused on confirmation of other kinases belonging to extracellular regulated signal kinases (Erk), in order to enhance 
the role of this pathway as one of the possible pathways of tumorigenesis in GG. Although Erk kinases are not uniformly activated in all cases, alternative (non-classic) Erk activation may explain lack or weak activation of Mek, in spite of the presence of active Erk $[14,16]$. This intriguing finding is still unclear.

Neurogenesis of GG remains uncertain. The most recent data reveal an immunohistochemical correlation between BRAF V600E mutation and expression of synaptophysin in the tumor cells, presence of dysplastic neurons and lymphocytic cuffs. However, the same study found no significant association between the presence of phospho Erk and BRAF V600E mutation in GG. Furthermore, the authors stated that the presence of BRAF mutation may be more likely in ganglionic elements of GG, although the mutation was also observed in glial cells of the tumor [17]. In our study we were unable to distinguish glial and ganglionic components of GG; therefore detailed pathological findings in GG subpopulations need further investigation.

To conclude, both Akt and Erk pathways take part in upregulation of mTOR in GG. In case of increased mTOR activation, these pathways may trigger uncontrolled proliferation and result in carcinogenesis. In the current study we argue that activation of the mTOR pathway, confirmed in GG samples, may result from potentiation of both Akt and Erk signaling.

\section{Acknowledgements}

This study was supported by the grant NR13001106/2009 from the Polish National Center of Research and Development.

\section{References}

1. Aronica E, Boer K, Doorn KJ, Zurolo E, Spliet WG, van Rijen PC, Baayen JC, Gorter JA, Jeromin A. Expression and localization of voltage dependent potassium channel Kv4.2 in epilepsy associated focal lesions. Neurobiol Dis 2009; 36: 81-95.

2. Astrinidis A, Henske EP. Tuberous sclerosis complex: linking growth and energy signaling pathways with human disease. Oncogene 2005; 24: 7475-7481.

3. Blümcke I, Giencke K, Wardelmann E, Beyenburg S, Kral T, Sarioglu N, Pietsch T, Wolf HK, Schramm J, Elger CE, Wiestler OD. The CD34 epitope is expressed in neoplastic and malformative lesions associated with chronic, focal epilepsies. Acta Neuropathol 1999; 97: 481-490.

4. Blümcke I, Wiestler OD. Gangliogliomas: an intriguing tumor entity associated with focal epilepsies. J Neuropathol Exp Neurol 2002; 61: 575-584.
5. Boer K, Troost D, Timmermans W, van Rijen PC, Spliet WG, Aronica E. Pi3K-mTOR signaling and AMOG expression in epilepsy-associated glioneuronal tumors. Brain Pathol 2010; 20 : 234-244.

6. Chandrashekhar TN, Mahadevan A, Vani S, Yasha TC, Sampath S, Chandramouli BA, Devi BI, Arvinda HR, Shankar SK. Pathological spectrum of neuronal/glioneuronal tumors from a tertiary referral neurological Institute. Neuropathology 2012; 32: 1-12.

7. Crino PB. Focal brain malformations: a spectrum of disorders along the mTOR cascade. Novartis Found Symp 2007; 288: 260272.

8. Curatolo P, Moavero R. mTOR inhibitors as a new therapeutic option for epilepsy. Expert Rev Neurother 2013; 13: 627-638.

9. Dan HC, Sun M, Yang L, Feldman RI, Sui XM, Ou CC, Nellist M, Yeung RS, Halley DJ, Nicosia SV, Pledger WJ, Cheng JQ. Phosphatidylinositol 3-kinase/Akt pathway regulates tuberous sclerosis tumor suppressor complex by phosphorylation of tuberin. J Biol Chem 2002; 277: 35364-35370.

10. Deb P, Sharma MC, Tripathi M, Sarat Chandra P, Gupta A, Sarkar C. Expression of CD34 as a novel marker for glioneuronal lesions associated with chronic intractable epilepsy. Neuropathol Appl Neurobiol 2006; 32: 461-468.

11. Gelabert-González M, Amo JM, Arcos Algaba A, Serramito García R, Castro Bouzas D, Díaz Cabana L, Prieto González A, Aran Echabe E, Bandín Diéguez FJ, Villa Fernández J, García Allut A. Intracranial gangliogliomas. A review of a series of 20 patients. Neurologia 2011; 26: 405-415.

12. Hay N, Sonenberg N. Upstream and downstream of mTOR. Genes Dev 2004; 18: 1926-1945.

13. Holmes GL, Stafstrom CE; Tuberous Sclerosis Study Group. Tuberous sclerosis complex and epilepsy: recent developments and future challenges. Epilepsia 2007; 48: 617-630.

14. Jozwiak J, Jozwiak S, Wlodarski P. Possible mechanisms of disease development in tuberous sclerosis. Lancet Oncol 2008; 9 : 73-79.

15. Jóźwiak J, Bikowska B, Grajkowska W, Sontowska I, Roszkowski M, Galus R. Activation of Akt/mTOR pathway in a patient with atypical teratoid/rhabdoid tumor. Folia Neuropathol 2010; 48: 185-189.

16. Kinkl N, Sahel J, Hicks D. Alternate FGF2-ERK1/2 signaling pathways in retinal photoreceptor and glial cells in vitro. J Biol Chem 2001; 276: 43871-43878.

17. Koelsche C, Wöhrer A, Jeibmann A, Schittenhelm J, Schinder G, Preusser M, Lasitschka F, von Deimling A, Capper D. Mutant BRAF V600E protein in ganglioglioma is predominantly expressed by neuronal tumor cells. Acta Neuropathol 2013; 125: 891-900.

18. Louis DN, Ohgaki H, Wiestler OD, Cavenee WK, Burger PC, Jouvet A, Scheithauer BW, Kleihues P. The 2007 WHO classification of tumours of the central nervous system. Acta Neuropathol 2007; 114: 97-109.

19. Majores M, Schick V, Engels G, Fassunke J, Elger CE, Schramm J, Blümcke I, Becker AJ. Mutational and immunohistochemical analysis of ezrin-, radixin-, moesin (ERM) molecules in epilepsy-associated glioneuronal lesions. Acta Neuropathol 2005; 110: 537-546. 
20. McLendon RE, Provenzale J. Glioneuronal tumors of the central nervous system. Brain Tumor Pathol 2002; 19: 51-58.

21. Miller DC, Koslow M, Budzilovich GN, Burstein DE. Synaptophysin: a sensitive and specific marker for ganglion cells in central nervous system neoplasms. Hum Pathol 1990; 21: 271-276.

22. Prayson RA. Tumours arising in the setting of paediatric chronic epilepsy. Pathology 2010; 42: 426-431.

23. Quinn B. Synaptophysin staining in normal brain: importance for diagnosis of ganglioglioma. Am J Surg Pathol 1998; 22: 550556.

24. Schick V, Majores M, Koch A, Elger CE, Schramm J, Urbach $H$, Becker AJ. Alterations of phosphatidylinositol 3-kinase pathway components in epilepsy-associated glioneuronal lesions. Epilepsia 2007; 48 Suppl 5: 65-73.

25. Yang I, Chang EF, Han SJ, Barry JJ, Fang S, Tihan T, Barbaro NM Parsa AT. Early surgical intervention in adult patients with ganglioglioma is associated with improved clinical seizure outcomes. J Clin Neurosci 2011; 18: 29-33. 